\title{
Validación de una escala para evaluar factores vinculados a la adherencia terapéutica en pacientes oncológicos ${ }^{*}$
}

\section{Validation of a scale to assess factors related to therapeutic adherence in cancer patients}

Recibido: septiembre 6 de 2009 | Revisado: marzo 10 de 2010 | Aceptado: octubre 8 de 2010

\author{
AlFONSO URZÚA M. ${ }^{* *}$ \\ Angelina Marmolejo C. \\ CATHERINe BARr D. \\ Escuela de Psicología, \\ Universidad Católica del Norte, Chile
}

\section{RESUMEN}

El objetivo del presente estudio fue construir una escala que evaluara factores vinculados a la adherencia terapéutica en pacientes oncológicos y evaluar sus propiedades psicométricas. A la muestra conformada por 120 participantes provenientes del Servicio de Oncología del Hospital Clínico Regional de Antofagasta, se aplicó el cuestionario creado y validado previamente en una muestra piloto en similar tipo de pacientes. Se analizó la consistencia interna y la estructura factorial del cuestionario, así como la relación entre las dimensiones evaluadas. De acuerdo a los resultados, la escala presenta un alfa superior a 0.9 , tanto a nivel total como en las diversas dimensiones, y una estructura factorial consistente con los factores teóricos elegidos. La conclusión de la investigación es que la escala construida puede constituirse en una herramienta de utilidad pronóstica de uso en pacientes oncológicos. Palabras clave autores

Adherencia terapéutica, cáncer, validez, confiabilidad.

Palabras clave descriptores

Pruebas psicológicas, psicometría, investigación cuantitativa, psicología de la salud.

\section{A B S T R A C T}

The aim of this study was to build and evaluate the psychometric properties of a scale to assess factors related to adherence in patients with cancer. In a sample of 120 patients from the Oncology Service from Hospital Clínico Regional de Antofagasta, we applied the questionnaire developed and previously validated in a pilot sample in similar patients. The internal consistency and factor structure of the questionnaire, and the relationship between the dimensions was assessed. According to the results, the scale has an alpha greater than 0.9 , both overall and in various dimensions, and a factor structure consistent with the theoretical factors chosen. The research concluded that the scale is constructed can become a useful prognostic tool for use in cancer patients.

Key words authors

Adherence, cancer, validity, reliability.

Key words plus

Psychological tests, psychometry, quantitative research, health psychology. 


\section{Introducción}

El cáncer es la segunda causa de muerte a nivel mundial. En Chile se estima que es responsable de cerca de un $22 \%$ de las muertes en el año, falleciendo por esta causa diariamente aproximadamente 48 personas (Vallebouna, 2001). Pese a estos datos la asociación entre cáncer y muerte ha ido quedando gradualmente atrás, dados los importantes avances que ha logrado la medicina actual en la efectividad de los diversos tratamientos. Esto ha permitido una mayor tasa de sobrevida en los pacientes que se adhieren al tratamiento indicado.

Independientemente del tipo de enfermedad con que sea diagnosticada una persona, la no adhesión a un determinado tratamiento conlleva múltiples consecuencias tanto para el paciente en los ámbitos personal, familiar, laboral y social, como para el sistema médico en general, provocando una serie de secuelas negativas, las que podrían incluso incidir en el progreso o agudización del cuadro clínico del paciente.

En países desarrollados, la adherencia terapéutica en pacientes que padecen enfermedades crónicas es cercana al 50 \% (Martín \& Grau, 2004). Además un $40 \%$ de los pacientes no cumple con las recomendaciones terapéuticas.

En el caso específico del cáncer, las particularidades de la enfermedad, como lo son la presencia de dolor en las etapas terminales, los efectos secundarios derivados de los tratamientos o bien los cambios de hábitos en la vida cotidiana, inciden en la existencia de una mayor probabilidad de que las personas opten por no seguir el tratamiento médico, fundamentalmente dado el carácter mayoritariamente ambulatorio de las intervenciones.

Pese a esto, son pocos los estudios que incorporan aspectos no biomédicos, tales como el costo físico, psicológico y emocional derivado de los efectos secundarios de los tratamientos, la aparición y permanencia de una serie de creencias respecto de los resultados de la enfermedad, sus diferentes formas de tratamiento o de los síntomas asociados, elementos todos que inciden en el pronóstico de la adherencia al tratamiento.
La adherencia terapéutica puede ser conceptualizada como la estrategia que permite que el paciente se mantenga y continúe el tratamiento y, de esta manera, logre cambios significativos en su comportamiento que mejoren su vida o, según la definición de la Organización Mundial de la Salud (OMS, 2004), como el grado en el cual el comportamiento del paciente, la toma de medicamentos, la dieta y la introducción de los cambios en sus estilos de vida responden a las indicaciones o recomendaciones dadas por el profesional de la salud.

Durante los últimos años se han realizado diversos estudios con la finalidad de determinar los factores o motivos que inciden en que un paciente siga o no las indicaciones médicas, sin embargo, los resultados que se han obtenido no han sido concluyentes respecto a su determinación.

Diversos autores han reportado factores similares, sin embargo la principal diferencia entre uno y otro radica en la categorización que hacen de cada uno de ellos. Por ejemplo, Ortiz y Ortiz (2005) agruparon las variables en tres tipos: psicológicas, demográficas y de comportamiento del paciente; a su vez Amigó, Fernández y Pérez, (2003) establecieron cuatro grupos de variables: las variables de la enfermedad, las variables del tratamiento, las variables médico-pacientes y, por último, las variables propias del paciente.

Si bien se puede señalar que los factores que inciden en la adherencia terapéutica son similares en la mayoría de las enfermedades, se han realizado diversos estudios para sistematizar los que podrían influir en la adhesión del paciente diagnosticado con cáncer, identificándose elementos que serían influyentes a la hora de seguir o no un tratamiento médico, tales como la naturaleza del régimen, su duración, complejidad, efectos secundarios, costosbeneficios, consecuencias y la satisfacción del paciente (Macià \& Méndez, 1999; Rodríguez-Marín, 1995).

Amigó et al. (2003) plantean un modelo basado en cuatro factores, a saber: (a) Variables Internas del Paciente: basados en los recursos, el conocimiento, las actitudes, las creencias, las percepciones y las expectativas del paciente; (b) Relación MédicoPaciente: relativos principalmente a lo que plantea 
la OMS, en lo referente a que una mejor relación entre proveedor y paciente podría mejorar la adherencia de los pacientes; (c) Proceso EnfermedadTratamiento: relacionado principalmente con la participación activa del paciente en el proceso de enfermedad, es decir, en cómo se involucra en este proceso y, de alguna manera, cómo influye en su mejoría o recuperación y (d) Costos- Beneficios de la Enfermedad: relacionado principalmente con las características propias de la enfermedad y a su vez del tratamiento, como es su complejidad, el tiempo de duración, los costos sociales, laborales y familiares, entre otros, que pueden estar asociados a esta patología y que claramente pueden incidir en la adherencia terapéutica.

Pese a lo fundamental que resulta el conocer la probabilidad que tiene un paciente de adherirse al tratamiento, considerando que esto es la clave del éxito en los programas de intervención en salud en enfermedades crónicas (Holguín, Correa, Arrivillaga, Cáceres \& Varela, 2006), los estudios en esta línea aún son escasos.

Se han realizado diversos intentos para medir los niveles de adherencia terapéutica, como por ejemplo, a través del "registro de huellas", esto es, del conteo o medición de las cantidades restantes de medicamento, a través del autorreporte, el monitoreo directo en sangre y orina de la sustancia, de sus metabolitos, de marcadores agregados o del uso de monitores de la medicación (Ehrenzweig, 2007; Macià \& Méndez, 1999; Rodríguez-Marín, 1995). Sin embargo, estas técnicas evalúan exclusivamente el cumplimiento del tratamiento y su componente medicamentoso, no aquellas indicaciones que se refieren a cambios de hábitos o estilos de vida (Martín \& Grau, 2004).

En este contexto, para los diferentes investigadores que han trabajado con el tema de la adherencia terapéutica, ha sido bastante complejo elaborar un instrumento que permita evaluar el nivel de adherencia de los pacientes, que incluya tanto una dimensión biológica como una psicológica o conductual. Este marco fundamenta la importancia de contar con un instrumento que permitiese evaluar o medir las probabilidades que tiene un paciente, diagnosticado con cáncer, de adherirse al trata- miento prescrito por el equipo medico tratante, instrumento que aún no ha sido reportado en la literatura revisada.

El contar con esta herramienta posibilitaría la incorporación temprana del paciente a un programa de apoyo psicológico o de modificación conductual, que permitiese cambiar las causales de la no adherencia y aumentar la probabilidad de efectividad y eficacia de las intervenciones médicas y psicológicas.

Con base en lo mencionado, el objetivo de esta investigación fue construir y validar una escala que permita evaluar los factores vinculados a la adherencia terapéutica en este tipo de pacientes y que permita aportar con información útil a los clínicos en el trabajo con aquellos que lo necesiten.

\section{Método}

Se diseñó una investigación en dos fases, la primera tendiente a la construcción del instrumento y en la segunda se evaluaron las propiedades psicométricas del instrumento diseñado.

\section{Participantes}

\section{Primera fase}

Se consideró una muestra piloto al azar, constituida por 40 pacientes ambulatorios que acudían al Servicio de Oncología del Hospital Clínico Regional de Antofagasta, que accedieron a participar en el estudio. El criterio de inclusión utilizado en esta etapa de la investigación fue que los participantes padecieran cáncer, indistintamente del tipo y de la etapa en que se encontrara la enfermedad. La edad promedio de este grupo fue de 49.7 años y aproximadamente el $70 \%$ eran mujeres.

\section{Segunda fase}

Para evaluar las propiedades psicométricas del instrumento se obtuvo una muestra no probabilística e intencional de 120 personas, con las mismas características de selección e inclusión que la muestra de la primera fase. De estas 120 personas 48 eran 
hombres (40 \%) y 72 , mujeres (60\%). El promedio de edad fue de 51.9 años $(D E=14.48)$, con una edad mínima de 23 años, una máxima de 83 años y una moda de 39 años.

En lo que respecta al tipo de cáncer, el más prevalente en esta muestra fue el cáncer de mama ( 30 casos), aunque este es casi exclusivo de la población femenina, por lo tanto en términos más estrictos y sin distinción de sexo, se puede señalar que aquel con mayor incidencia en esta investigación es el cáncer de pulmón (15 casos).

En cuanto a los tipos de tratamiento, la mayoría se encuentra en proceso de quimioterapia (63 casos) constituyéndose en un $52.5 \%$ del total de la muestra, seguido por la cirugía (15 casos), radioterapia (14 casos) y tratamiento combinado de quimioterapia con radioterapia (13 casos).

Por último, en cuanto a la etapa de la enfermedad en la que se encuentran los pacientes participantes del estudio, se puede señalar que 44 participantes $(36.7 \%)$ se encontraban en etapa avanzada o terminal (Etapa IV), 26 (21.7 \%) en etapa III, 40 $(33.3 \%)$ en etapa II y 10 personas $(8.3 \%)$ en la etapa inicial de la enfermedad (Etapa I).

\section{Procedimientos}

\section{Primera fase}

La construcción del instrumento se llevó a cabo en diversas etapas. La primera etapa consistió en una revisión bibliográfica sobre los términos adherencia y cáncer, tanto en español como en inglés. En esta revisión se buscaba conocer los factores reportados por la literatura como incidentes en los que los pacientes se adhieran o no a un tratamiento. Tomando como base dicha revisión, se pasó a una segunda etapa, la cual consistió en la elaboración de un marco teórico que integrase las diversas variables mencionadas en la literatura y que daría el sustento a la elección de las diversas preguntas (este material puede ser solicitado a los autores). Una tercera etapa consistió en la generación de una serie de aseveraciones tentativas, mediante la técnica conocida como "lluvia de ideas". Esto posibilitó obtener un listado de aproximadamente 200 reactivos, de entre los cuales fueron elegidos 100 que constituirían la escala piloto (también puede ser solicitado a los autores).

La cuarta etapa de esta primera fase constituye el proceso de obtención de permisos y recolección de datos en una muestra piloto. Para esto, se solicitó autorización al jefe del Servicio de Oncología Ambulatoria para llevar a cabo la aplicación de la escala con los pacientes oncológicos del servicio, explicándosele los objetivos y fundamentos de este estudio. Una vez obtenida la autorización, se procedió a contactar a los pacientes de forma aleatoria, hasta lograr completar un total de 40 personas, a las cuales se les solicitó su consentimiento informado y se les explicó los principales objetivos del estudio.

Posteriormente, se procedió a la aplicación de la escala, lo que tuvo que realizarse de forma asistida, es decir, fue necesario que los evaluadores leyeran una a una las preguntas de la escala para que de tal forma el paciente pudiera responder de forma verbal a la pregunta, proceso que demoraba alrededor de 20 minutos. Una vez terminado el proceso de aplicación piloto, los datos se ingresaron en una base de datos construida en SPSS 11.5.

El sexto paso de la primera fase lo constituyeron los análisis estadísticos iniciales, a fin de determinar cuáles serían los factores que compondrían definitivamente la escala, además de los reactivos que conformarían dicho instrumento.

Fueron invertidos aquellos reactivos cuya valencia era negativa a fin de que todos los reactivos apuntaran a un solo sentido. Los datos recogidos fueron sometidos a un análisis de correlación ítem con el total del test menos el ítem. Se decidió eliminar todos aquellos reactivos cuya correlación ítem-test fuese menor a 0.30 , eliminándose de esta manera 45 preguntas. Los datos restantes fueron sometidos a un análisis factorial exploratorio mediante el método de extracción de componentes principales con rotación Varimax. En esta etapa se eliminaron los reactivos que tenían pesos factoriales similares en diversos factores y aquellos que no se ajustaban teóricamente al factor o la dimensión en que tenían mayor peso factorial.

Se llega así a una solución factorial de 20 reactivos agrupados en tres dimensiones con autovalores 
mayores que 1, los que explican un $73.31 \%$ de la varianza (puede ser solicitado a los autores), con comunalidades que van entre 0.51 y 0.85 . La medida de adecuación muestral de Kaiser-Meyer-Olkin fue de 0.82. La prueba de esfericidad de Bartlett permite rechazar la hipótesis de igualdad de las matrices $\left(\chi^{2}(190)=761.098 ; p=0.00\right)$. El correlato teórico de esta distribución factorial puede ser solicitado a los autores.

Segunda fase

Luego de haber obtenido el instrumento, se comenzó el proceso de aplicación. Con esta finalidad se contactó a un total aproximado de 220 personas, de similares características a los que participaron en la aplicación piloto. De estas, aceptaron participar 120, ya que las demás personas, manifestaron diversas razones por las cuales no participarían.

De igual manera, se solicitó a cada uno de los sujetos su consentimiento informado, explicándoles los objetivos de esta investigación e indicándoles las instrucciones básicas para responder la encuesta.

Del total de personas que participaron de este estudio, a un $60 \%$ se les aplicó la escala de forma asistida. En cuanto al 40 \% restante, su aplicación fue autoadministrada y el tiempo que ocupó cada paciente para completarla, fluctuó entre 10 y 15 minutos.

Luego que las encuestas fueron aplicadas, los datos fueron ingresados a una base de datos construida utilizando el software del paquete estadístico para ciencias sociales, SPSS 11.5, para su posterior análisis.

\section{Instrumento}

La escala final está conformada por un total de 20 reactivos. El formato de respuesta es tipo Likert con valores que oscilan de 1 a 4 , dependiendo del grado de acuerdo o desacuerdo con las aseveraciones propuestas, en donde el valor 1 equivale a totalmente en desacuerdo y el valor 4 equivale a totalmente de acuerdo, teniendo presente que sus valores intermedios, con valores 2 y 3 , significan desacuerdo y de acuerdo, respectivamente.
Las dimensiones que finalmente componen la escala de adherencia terapéutica son: (a) Expectativas y herramientas personales para enfrentar la enfermedad (diez reactivos), (b) Creencias sobre el tratamiento (seis reactivos) y (c) Efectos percibidos del tratamiento (cuatro reactivos).

A la escala se le adicionaron además algunas de las variables sociodemográficas que se consideraron necesarias para posteriores análisis como son: edad, sexo, tipo de tratamiento y etapa en la que se encuentra la enfermedad.

\section{Análisis estadístico}

En ambas fases, se realizaron análisis de la consistencia interna a través del estadístico a de Cronbach. Asimismo, en ambas fases se calculó la correlación ítem-total menos el ítem e ítem dimensión. En ambas fases se realizaron análisis factoriales exploratorios, a través del método análisis de componentes principales con rotación por el método normalización Varimax con Kaiser. A la escala final se le realizó un análisis factorial confirmatorio mediante el programa Lisrel 8.30.

\section{Resultados}

\section{Fiabilidad}

El $\alpha$ de Cronbach obtuvo los siguientes valores: Factor 1 (10 reactivos) $=0.9$; Factor 2 (4 reactivos) $=0.93 ;$ Factor 3 (6 reactivos $)=0.91$. La escala total obtuvo un alfa de 0.96 .

\section{Análisis de ítems}

Con excepción de la pregunta nueve (creencia en Dios), que no correlaciona con las preguntas 13 y 14 (manejo información), los reactivos están correlacionados significativamente entre ellos. En general, todos los reactivos presentan una correlación superior a 0.6, tanto con la escala total como con su respectiva dimensión, con excepción de las preguntas 13 y 14 (manejo información), que presentan un valor inferior a 0.5 (Tabla 1). 
TABLA 1

Correlaciones ítem-total e item-dimensión

\begin{tabular}{|c|c|c|c|}
\hline & Reactivo & Ítem-total & Ítem-dimensión \\
\hline 1 & Creo que el tratamiento me dará buenos resultados. & 0.8715 & 0.8166 \\
\hline 2 & Pienso que puedo luchar contra la enfermedad. & 0.7992 & 0.7926 \\
\hline 3 & Tengo la fuerza para salir adelante con mi enfermedad. & 0.8241 & 0.8161 \\
\hline 4 & El tratamiento me ayudara a mejorar. & 0.8724 & 0.8713 \\
\hline 5 & Tengo las herramientas para salir adelante con la enfermedad. & 0.6672 & 0.6848 \\
\hline 6 & Cuando me interesa saber de mi enfermedad, pregunto. & 0.6767 & 0.7199 \\
\hline 7 & Sé que puedo seguir adelante con mi tratamiento. & 0.7864 & 0.7102 \\
\hline 8 & Sé que tengo las habilidades para colaborar con mi tratamiento. & 0.7915 & 0.7958 \\
\hline 9 & Confío en que Dios me ayudara a salir adelante. & 0.6086 & 0.6069 \\
\hline 10 & Sé que Dios me entrega la fuerza necesaria para superar la enfermedad. & 0.5854 & 0.5773 \\
\hline 11 & Siento que puedo superar la enfermedad. & 0.7502 & 0.685 \\
\hline 12 & Creo que puedo superar la enfermedad. & 0.7898 & 0.7304 \\
\hline 13 & Manejo información respecto de lo que significa vivir con cáncer. & 0.4246 & 0.4929 \\
\hline 14 & $\begin{array}{l}\text { Manejo información respecto de las consecuencias de seguir o no un } \\
\text { tratamiento. }\end{array}$ & 0.435 & 0.5039 \\
\hline 15 & Desde que comencé el tratamiento, todo ha ido mejorando. & 0.7992 & 0.8248 \\
\hline 16 & Pienso que el tratamiento ha hecho que los síntomas disminuyan. & 0.6758 & 0.81 \\
\hline 17 & Me he sentido mejor desde que empecé el tratamiento. & 0.6962 & 0.8906 \\
\hline 18 & Cada día me siento un poco mejor. & 0.6155 & 0.7877 \\
\hline 19 & $\begin{array}{l}\text { Siento que a pesar de los efectos secundarios, es mejor continuar el } \\
\text { tratamiento. }\end{array}$ & 0.628 & 0.594 \\
\hline 20 & Sé que puedo seguir adelante con mi tratamiento. & 0.7012 & 0.6651 \\
\hline
\end{tabular}

Fuente: elaboración propia.

\section{Estructura factorial}

La medida de adecuación muestral de KaiserMeyer-Olkin es de 0.89 , lo que posibilita realizar el análisis factorial. La prueba de esfericidad de Bartlett rechaza que ambas matrices sean iguales, existiendo, por tanto, correlaciones entre los datos $\left(\chi^{2}(190)=2430.129 ; p=0.00\right)$.

El análisis factorial exploratorio con método de rotación de normalización Varimax con Kaiser, aporta un modelo de cuatro factores con autovalores mayores que 1 que explican un $77.98 \%$ de la varianza. Las comunalidades oscilan entre 0.54 y 0.95 (la tabla que contiene los pesos factoriales de cada uno de los reactivos en el correspondiente factor, puede ser solicitada a los autores).

Dado que la estructura factorial es distinta a la obtenida en la fase 1 de construcción del instru- mento, y fundamentalmente que no se observa un adecuado ajuste teórico de las variables en dos de los factores encontrados, se realizó un nuevo análisis exploratorio forzando los datos a una salida de tres factores, de acuerdo a lo esperado teóricamente.

La nueva matriz de distribución, en que los tres factores explican un $71.71 \%$ de la varianza, puede ser observada en la Tabla 2.

Dado que esta estructura factorial fue la más adecuada en términos de su correlato teórico (Tabla 3), se calculó nuevamente con base en esta configuración tanto la consistencia interna como la correlación ítem-dimensión, a fin de compararlas con la distribución teórica inicial derivada de la primera fase.

La consistencia interna del primer factor, denominado Creencias sobre tratamiento y enfermedad, compuesto por 13 reactivos, presentó un alfa 
TABLA 2

Matriz de componentes rotados solución de tres factores

\begin{tabular}{|c|c|c|c|c|}
\hline & & 1 & 2 & 3 \\
\hline 1 & Creo que el tratamiento me dará buenos resultados. & 0.772 & 0.429 & 0.201 \\
\hline 2 & Pienso que puedo luchar contra la enfermedad. & 0.645 & 0.544 & 0.068 \\
\hline 3 & Tengo la fuerza para salir adelante con mi enfermedad. & 0.755 & 0.398 & 0.158 \\
\hline 4 & El tratamiento me ayudara a mejorar. & 0.806 & 0.388 & 0.202 \\
\hline 5 & Tengo las herramientas para salir adelante con la enfermedad. & 0.665 & 0.236 & 0.232 \\
\hline 6 & Cuando me interesa saber de mi enfermedad, pregunto. & 0.706 & 0.113 & 0.39 \\
\hline 7 & Sé que puedo seguir adelante con mi tratamiento. & 0.626 & 0.526 & 0.12 \\
\hline 8 & Sé que tengo las habilidades para colaborar con mi tratamiento. & 0.73 & 0.333 & 0.257 \\
\hline 9 & Confío en que Dios me ayudara a salir adelante. & 0.763 & 0.152 & -0.075 \\
\hline 10 & Sé que Dios me entrega la fuerza necesaria para superar la enfermedad. & 0.73 & 0.121 & 0.001 \\
\hline 11 & Siento que puedo superar la enfermedad. & 0.644 & 0.436 & 0.137 \\
\hline 12 & Creo que puedo superar la enfermedad. & 0.604 & 0.506 & 0.218 \\
\hline 19 & $\begin{array}{l}\text { Siento que a pesar de los efectos secundarios, es mejor continuar el } \\
\text { tratamiento. }\end{array}$ & 0.605 & 0.243 & 0.228 \\
\hline 15 & Desde que comencé el tratamiento, todo ha ido mejorando. & 0.43 & 0.764 & 0.166 \\
\hline 16 & Pienso que el tratamiento ha hecho que los síntomas disminuyan. & 0.292 & 0.802 & 0.039 \\
\hline 17 & Me he sentido mejor desde que empecé el tratamiento. & 0.255 & 0.877 & 0.053 \\
\hline 18 & Cada día me siento un poco mejor. & 0.114 & 0.894 & 0.129 \\
\hline 20 & Sé que puedo seguir adelante con mi tratamiento. & 0.485 & 0.59 & 0.067 \\
\hline 13 & Manejo información respecto de lo que significa vivir con cáncer. & 0.171 & 0.109 & 0.945 \\
\hline 14 & $\begin{array}{l}\text { Manejo información respecto de las consecuencias de seguir o no un } \\
\text { tratamiento. }\end{array}$ & 0.182 & 0.104 & 0.956 \\
\hline
\end{tabular}

Nota. Método de extracción: Análisis de componentes principales. Método de rotación: Normalización Varimax con Kaiser. La rotación ha convergido en 5 iteraciones.

Fuente: elaboración propia.

de 0.95. El segundo factor, denominado Efectos percibidos del tratamiento, está compuesto por cinco reactivos y obtuvo un alfa de 0.92 . La tercera dimensión denominada Manejo de Información, quedó constituida por solo dos reactivos y presentó un alfa de 0.97 .

Tal como se puede observar en la Tabla 4, la correlación ítem-dimensión es mayoritariamente superior en esta nueva solución, incluso en aquellos que en la distribución inicial eran inferiores a 0.5 (reactivos 13 y 14).
Al realizar un análisis factorial confirmatorio se encuentra que si bien los valores no son los esperados, se aproximan al valor ideal (0.9), y en el caso del RMSEA (0.08), revelando un ajuste moderado de los datos a la propuesta teórica de tres factores (Tabla 5).

\section{Discusión}

La escala cumple con las propiedades psicométricas adecuadas para ser utilizada como una medida de 
tamizaje, destinada a la pesquisa precoz de factores de riesgo en la no adherencia terapéutica.

Los valores de consistencia interna obtenidos permiten sugerir su uso tanto a nivel de prueba de tamizaje como también de indicador o medida de conducta en salud individual (Scientific Advisory Committee of the Medical Outcomes Trust, 2002).
La validez de constructo, dada por la estructura factorial de la escala, es asimismo congruente con la evidencia teórica al respecto. Los tres factores propuestos: Creencias sobre el tratamiento y la enfermedad, Efectos percibidos del tratamiento y Manejo de información cuentan con un amplio respaldo teórico que avala su inclusión como factores

TABLA 3

Correlato teórico de la solución factorial de 20 reactivos agrupados en tres dimensiones

\begin{tabular}{|c|c|c|c|}
\hline FACTOR / DIMENSIÓN & DESCRIPCIÓN DE DIMENSIÓN & SUBDIMENSIÓN & REACTIVOS \\
\hline $\begin{array}{l}\text { 1. Variables Internas del } \\
\text { Paciente (Creencias del } \\
\text { paciente asociada a su en- } \\
\text { fermedad). }\end{array}$ & $\begin{array}{l}\text { Estas variables se relacionan con las } \\
\text { características internas, o de perso- } \\
\text { nalidad, del paciente. Orientadas } \\
\text { principalmente a los estilos de afron- } \\
\text { tamiento, modelos de creencias, } \\
\text { estilos de vida y formas de resolver } \\
\text { conflictos. }\end{array}$ & $\begin{array}{l}\text { Expectativas del pa- } \\
\text { ciente. } \\
\text { Creencias acerca de la } \\
\text { causa de la enferme- } \\
\text { dad. } \\
\text { Creencias acerca del } \\
\text { curso de la enfermedad. } \\
\text { Motivación. } \\
\text { Estrategias de afronta- } \\
\text { miento. } \\
\text { Significación de la en- } \\
\text { fermedad. } \\
\text { Expectativas de auto } \\
\text { eficacia. }\end{array}$ & $\begin{array}{l}\text { 1. Creo que el trata- } \\
\text { miento me dará buenos } \\
\text { resultados. } \\
\text { 2. Pienso que puedo } \\
\text { luchar contra la enfer- } \\
\text { medad. } \\
\text { 3. Tengo la fuerza para } \\
\text { salir adelante con mi } \\
\text { enfermedad. } \\
\text { 4. El tratamiento me } \\
\text { ayudará a mejorar. } \\
\text { 5. Tengo las herramien- } \\
\text { tas para salir adelante } \\
\text { con la enfermedad. } \\
\text { 6. Cuando me interesa } \\
\text { saber acerca de mi en- } \\
\text { fermedad, pregunto. } \\
\text { 7. Sé que puedo seguir } \\
\text { adelante con mi trata- } \\
\text { miento. } \\
\text { 8. Sé que tengo las ha- } \\
\text { bilidades para colaborar } \\
\text { con mi tratamiento. } \\
\text { 9. Confío en que Dios } \\
\text { me ayudara a salir ade- } \\
\text { lante. } \\
\text { 10. Sé que Dios me en- } \\
\text { trega la fuerza necesaria } \\
\text { para superar la enfer- } \\
\text { medad. } \\
\text { 11. Siento que puedo } \\
\text { superar la enfermedad. } \\
\text { 12. Creo que puedo su- } \\
\text { perar la enfermedad. } \\
\text { 19. Siento que a pesar } \\
\text { de los efectos secunda- } \\
\text { rios es mejor continuar } \\
\text { el tratamiento. }\end{array}$ \\
\hline
\end{tabular}




\begin{tabular}{|c|c|c|c|}
\hline FACTOR / DIMENSIÓN & DESCRIPCIÓN DE DIMENSIÓN & SUBDIMENSIÓN & REACTIVOS \\
\hline $\begin{array}{l}\text { 2. Variable efecto percibido } \\
\text { del tratamiento. }\end{array}$ & $\begin{array}{l}\text { Estos factores están vinculados a las } \\
\text { características propias del tratamien- } \\
\text { to, es decir la complejidad de éste, el } \\
\text { tiempo de duración, los efectos se- } \\
\text { cundarios que provoca el tratamien- } \\
\text { to, además de un aspecto relevante } \\
\text { asociado al efecto que el paciente } \\
\text { percibe sobre su tratamiento. }\end{array}$ & $\begin{array}{l}\text { 2.1 Efectos secundarios } \\
\text { del tratamiento. } \\
\text { 2.2 Gastos involucra- } \\
\text { dos en el tratamiento. } \\
\text { 2.3 Relación costo- } \\
\text { beneficios del trata- } \\
\text { miento. }\end{array}$ & $\begin{array}{l}\text { 15. Desde que comencé } \\
\text { el tratamiento todo ha } \\
\text { ido mejorando. } \\
\text { 16. Pienso que el trata- } \\
\text { miento ha hecho que los } \\
\text { síntomas de la enferme- } \\
\text { dad disminuyan. } \\
\text { 17. Me he sentido mejor } \\
\text { desde que empecé el } \\
\text { tratamiento. } \\
\text { 18. Cada día me siento } \\
\text { un poco mejor. } \\
\text { 20. Sé que puedo seguir } \\
\text { adelante con mi trata- } \\
\text { miento. }\end{array}$ \\
\hline $\begin{array}{l}\text { 3. Variables Externas del } \\
\text { Paciente (Manejo de in- } \\
\text { formación por parte del } \\
\text { paciente). }\end{array}$ & $\begin{array}{l}\text { Estas variables están relacionadas } \\
\text { fundamentalmente con el contexto } \\
\text { en el cual se desenvuelve el paciente. } \\
\text { La cantidad de información que ma- } \\
\text { neja respecto de su enfermedad. }\end{array}$ & 3.1 Información. & $\begin{array}{l}\text { 13. Manejo información } \\
\text { respecto de lo que signi- } \\
\text { fica vivir con cáncer. } \\
14 \text {. Manejo información } \\
\text { respecto de las conse- } \\
\text { cuencias de seguir o no } \\
\text { un tratamiento. }\end{array}$ \\
\hline
\end{tabular}

Fuente: elaboración propia.

TABLA 4

Correlaciones item-dimensión en soluciones de tres factores inicial y final

\begin{tabular}{llcc}
\hline \multicolumn{1}{c}{ Reactivo } & \multicolumn{1}{c}{$\begin{array}{c}\text { Solución } \\
\text { inicial }\end{array}$} & $\begin{array}{c}\text { Solución } \\
\text { final }\end{array}$ \\
\hline 1 & Creo que el tratamiento me dará buenos resultados. & 0.8166 & 0.885 \\
2 & Pienso que puedo luchar contra la enfermedad. & 0.7926 & 0.8008 \\
3 & Tengo la fuerza para salir adelante con mi enfermedad. & 0.8161 & 0.8463 \\
4 & El tratamiento me ayudará a mejorar. & 0.8713 & 0.9068 \\
5 & Tengo las herramientas para salir adelante con la enfermedad. & 0.6848 & 0.7005 \\
6 & Cuando me interesa saber de mi enfermedad, pregunto. & 0.7199 & 0.6809 \\
7 & Sé que puedo seguir adelante con mi tratamiento. & 0.7102 & 0.7811 \\
8 & Sé que tengo las habilidades para colaborar con mi tratamiento. & 0.7958 & 0.8172 \\
9 & Confío en que Dios me ayudara a salir adelante. & 0.6069 & 0.639 \\
10 & Sé que Dios me entrega la fuerza necesaria para superar la enfermedad. & 0.5773 & 0.6018 \\
11 & Siento que puedo superar la enfermedad. & 0.685 & 0.7669 \\
12 & Creo que puedo superar la enfermedad. & 0.7304 & 0.7778 \\
13 & Manejo información respecto de lo que significa vivir con cáncer. & 0.4929 & 0.9462 \\
14 & Manejo información respecto de las consecuencias de seguir o no un tratamiento. & 0.5039 & 0.9462 \\
15 & Desde que comencé el tratamiento, todo ha ido mejorando. & 0.8248 & 0.8287 \\
16 & Pienso que el tratamiento ha hecho que los síntomas disminuyan. & 0.81 & 0.8204 \\
17 & Me he sentido mejor desde que empecé el tratamiento. & 0.8906 & 0.8728 \\
18 & Cada día me siento un poco mejor. & 0.7877 & 0.7809 \\
19 & Siento que a pesar de los efectos secundarios, es mejor continuar el tratamiento. & 0.594 & 0.6172 \\
20 & Sé que puedo seguir adelante con mi tratamiento. & 0.6651 & 0.6516 \\
\hline
\end{tabular}

Fuente: elaboración propia. 
TABLA 5

Índices de ajuste para el modelo factorial

\begin{tabular}{cccccc}
\hline$\chi^{2}$ & CFI & NFI & GFI & RFI & RMSEA \\
\hline 563.190 & 0.835 & 0.783 & 0.686 & 0.753 & 0.138 \\
\hline
\end{tabular}

$\chi^{2}=$ Normal Theory Weighted Least Squares Chi-Square; CFI = Comparative Fit Index; NFI = Normed Fit Index; GFI = Goodness of Fit Index; RFI = Relative Fit Index; RMSEA = Root Mean Square Error of Approximation.

Fuente: elaboración propia.

vinculados a la adherencia terapéutica en pacientes oncológicos.

El factor Creencias sobre tratamiento y enfermedad, incluye reactivos relacionados con las expectativas, creencias, motivación, pensamientos y sentimientos que el paciente tiene con relación a la enfermedad, su curso y el tratamiento. Esta dimensión es reportada en otras investigaciones con el nombre de Creencias de los pacientes (Martín \& Grau, 2004) o como variables internas del paciente (Amigó et al., 2003).

Estos aspectos internos del paciente poseen un gran valor a la hora de determinar su adherencia a un determinado tratamiento. Esto podría explicarse respecto de la motivación que este tenga en cuanto a su recuperación, sus estilos de afrontamiento, e incluso en cuanto a patrones socioculturales y familiares, los que se traspasan generacionalmente quedando arraigados en la cultura de las personas. En este sentido, se ha planteado que el hecho de que una persona adhiera o mantenga comportamientos saludables va a depender de sus creencias, las cuales podrían ser consideradas como los determinantes más próximos a las conductas de salud y van a depender de sistemas socioculturales de pertenencia y referencia de la persona (Ehrenzweig, 2007).

La dimensión denominada Efectos percibidos del tratamiento y la enfermedad, comprende lo que para el paciente sería importante evaluar sobre las eventuales consecuencias, negativas o positivas, que le significaría seguir este proceso, lo que claramente determinaría su adhesión o no al tratamiento. Este hecho es algo conocido tanto en el ámbito de la medicina como en el campo de la Psicología de la Salud, en donde los pacientes optan o desisten de un tratamiento, en gran medida debido a diversos factores extrínsecos relacionados con la enfermedad y/o con el tratamiento. Esto ha sido planteado similarmente por Friedman y DiMatteo (1989) quienes señalan que, dependiendo de las características propias del tratamiento, habría una mayor o menor adherencia; algunas de estas serían la naturaleza del régimen, su duración, complejidad, efectos secundarios, costos, beneficios, consecuencias y otros factores como la satisfacción del paciente. Bajo esta mirada, se asume que la conducta y las decisiones estarían basadas en un análisis subjetivo elaborado, costo-beneficios de los resultados probables (Ehrenzweig, 2007), en donde las personas optarían por maximizar la utilidad prefiriendo conductas asociadas con la mayor utilidad esperada (Conner \& Norman, 1996; Marteau, 1993).

Macià-Antón y Méndez (1999) plantean que los regímenes terapéuticos que provocan efectos secundarios pueden llegar a ser más perturbadores que los síntomas de la enfermedad, afectando así las tasas de adherencia; los posibles efectos colaterales de los medicamentos pueden actuar como estímulos aversivos y producir así un descenso en la adherencia.

Por lo tanto, sería importante para el paciente oncológico poder percibir como efectivo el tratamiento al cual está siendo sometido, lo que le permite evaluarlo como positivo, es decir, que está siendo eficaz en cuanto a controlar el progreso de la enfermedad y que, a su vez, los efectos secundarios o costos del tratamiento, sean vistos como menos relevantes, o al menos que no logren incidir en una posible deserción terapéutica. Sin embargo, estos son planteamientos que podrían ser contrastados en futuros estudios sobre adherencia en poblaciones similares a la de esta investigación, lo que permitiría avalar o refutar la idea recientemente expuesta, así como también el hecho de que con base en esto se 
podría esperar que en etapas más avanzadas del cáncer, debido principalmente a los síntomas físicos asociados, principalmente el dolor, los pacientes tenderían a aumentar su adherencia al tratamiento.

La tercera y última dimensión, denominada Manejo de información, hace referencia a la posibilidad que tienen los pacientes de acceder a información adecuada, pertinente y atingente respecto de su enfermedad y tratamiento. Tal como refieren Holguín et al. (2006), solo la información clara, suficiente, ajustada a las necesidades del paciente y oportuna va a contribuir a una implicación activa en el tratamiento.

Lo anterior implica, idealmente, que el acceso a la información debe ser modulado y entregado, por personal calificado y capacitado para esto, ya que la información debe estar orientada en función de las diversas características del paciente oncológico, a sus estilos de afrontamiento, a las percepciones subjetivas que tiene este acerca de su enfermedad, así como también de la concepción que tienen él y su familia respecto del cáncer.

En esta marco, Ginarte (2001) señala la importancia de una comunicación eficaz entre el profesional de la salud y el paciente, en donde el hecho de proporcionar la información necesaria de modo que favorezca y garantice niveles mínimos de compresión por parte del enfermo, contribuye a mejorar la adherencia, fomentando el traspaso paulatino de la responsabilidad al propio sujeto (Téllez, 2004).

Futuras investigaciones pudiesen salvar las limitaciones del presente estudio, contrastando los resultados obtenidos con este instrumento en poblaciones similares, pero con una muestra mayor; así como también evaluar posibles diferencias entre estos factores y variables propias de la enfermedad como por ejemplo la etapa de esta, los tipos de tratamientos o bien, con variables sociodemográficas como el sexo y la edad de los pacientes, pudiendo tal vez ampliar el espectro de conocimientos en esta área.

Tal como se ha planteado, la utilidad fundamental de la escala propuesta es brindar la posibilidad de detectar aquellas variables que intervienen en que efectivamente un paciente oncológico se adhiera a su tratamiento. Esto sería de gran importancia, ya que al evaluar y conocer parte de la multiplicidad de factores psicosociales intervinientes en el cumplimiento (Martín, 2003), se podría trabajar con ellos, de manera de potenciar e incentivar conductas dirigidas a aumentar la adherencia, a través de la incorporación temprana a un programa de apoyo psicológico, psicoeducación o procesos de acompañamiento, dirigidos tanto al propio paciente como a su familia, con el fin de modificar gradualmente las creencias, las expectativas, los prejuicios y la forma en que la familia oncológica percibe el proceso de enfermedad, y a su vez lo que significa adherirse al tratamiento.

\section{Referencias}

Amigó, I., Fernández, C. \& Pérez, M. (2003). Manual de psicología de la salud (2a. ed.). Madrid: Pirámide.

Conner, M. \& Norman, P. (1996). The role of social cognition models in predicting health behaviours: Future directions. En M. Conner \& P. Norman (Eds.), Predicting health behaviours. Research and practice with social cognition models (pp. 179-225). Buckingham, UK: Open University Press.

Ehrenzweig, Y. (2007). Modelos de cognición social y adherencia terapéutica en pacientes con cáncer. Avances en Psicología Latinoamericana, 25(1), 9 -13.

Friedman, H. \& DiMatteo, M. (1989). Adherence and practitioner patient relationship. En $\mathrm{H}$. Friedman \& M. DiMatteo (Eds.), Health Psychology (pp. 68100). New York: Prentice Hall.

Ginarte, Y. (2001). La adherencia terapéutica. Revista Cubana Médica, 17(5), 503-504.

Holguín, L., Correa, D., Arrivillaga, M., Cáceres, D. \& Varela, M. (2006). Adherencia al tratamiento de hipertension arterial: efectividad de un programa de intervencion biopsicosocial. Universitas Psychologica, 5(3), 535-547.

Macià-Antón, D. \& Méndez, F. (1999). Líneas actuales de investigación en psicología de la salud. En M. Simón (Ed.), Manual de psicología de la salud. Fundamentos, metodología y aplicaciones (pp. 217-256). Madrid: Biblioteca Nueva.

Marteau, T. (1993). Health beliefs and attributions. En A. K. Broome (Ed.), Health psychology. Processes and applications (pp. 1-24). Londres: Chapman \& Hall. 
Martín, L. (2003). Aplicaciones de la psicología en el proceso salud-enfermedad. Revista Cubana de Salud Pública, 29(3), 275-281.

Martín, L. \& Grau, J. (2004). La investigación de la adherencia terapéutica como un problema de la psicología de la salud. Psicología y Salud, 14(1), 89-99.

Organización Mundial de la Salud. (2004). Adherencia a los tratamientos a largo plazo: pruebas para la acción (Informe Técnico). Washington, DC: Ediciones Organización Panamericana de la Salud.

Ortiz, M. \& Ortiz, E. (2005). Adherencia al tratamiento en pacientes diabéticos tipo 1 chilenos: una aproximacion psicológica. Revista Médica de Chile, 133, 307-313.
Rodríguez-Marín, J. (1995). Psicología social de la salud. Madrid: Síntesis.

Scientific Advisory Committee of the Medical Outcomes Trust. (2002). Assessing health status and quality of life instruments: Attribute and review criteria. Quality of Life Research, 11, 193-205.

Téllez, A. (2004). Prevención y adherencia: dos claves para el enfrentamiento de las enfermedades crónicas. Revista Médica de Chile, 132, 1453-1455.

Vallebouna, C. (2001). Registros poblacionales de cáncer: avances en Chile. Departamento de Epidemiología. Ministerio de Salud, Chile. Recuperado el 15 de junio, 2009, de http://epi.minsal.cl/epi/html/ presenta/Taller2001/Regpobcan(Clelia\%20Ballevuona).ppt 